Methods In 2019, programme mentors identified factors outside the control of the intervention potentially affecting performance as reflected in the QoC data, including lack of oxygen concentrators, electricity and essential medicines. Alongside QOC measures, narrative data were recorded to test this. QOC data from one four-week period (May 2019) in four 'hub' regional hospitals were assessed against qualitative data. Frequency of UK mentor intervention was also recorded. Results Variability in QoC between hospitals corresponded with narrative findings. While 3 hubs showed 100\% correct oxygen prescription for SRD, one recorded 33\% corresponding with narrative reporting of issues with oxygen concentrators and electricity supply. The same hub recorded $8 \%$ appropriate blood transfusions, with narrative reporting of prescriptions managed by a practitioner without ETAT + experience; the other hubs reported 100\%. Need for external mentors to intervene e.g. for management of critically unwell children and intravenous cannulation varied from 20-79\%; a higher frequency of mentor intervention corresponded with higher QoC scores.

Discussion ETAT+ plausibly improves QOC in participating facilities in Sierra Leone. However it is important to observe factors outside of the intervention's direct control which may negatively affect results. Staff knowledge and performance may improve but remain constrained by poor equipment and infrastructure. Presence of external programme clinical mentors may affect results (with thresholds to intervene varying between clinicians). More detailed understanding of the hospital care 'ecology' is required to assess ETAT+'s impact on care quality and health outcomes.

\section{G449(P) USING THE MODIFIED DELPHI METHOD TO ESTABLISH CONSENSUS ON CLINICAL ALGORITHMS FOR A NEONATAL MOBILE HEALTH APPLICATION FOR LOW- INCOME COUNTRIES}

${ }^{1} \mathrm{M}$ Evans, ${ }^{1} \mathrm{C} C$ Crehan, ${ }^{2,3} \mathrm{M}$ Corden, ${ }^{1,4} \mathrm{M}$ Heys. ${ }^{1}$ Great Ormond Street Hospital Institute of Child Health, UCL, London, UK; ${ }^{2}$ Division of Hospital Medicine, Department of Pediatrics, Children's Hospital Los Angeles, Los Angeles, USA; ${ }^{3}$ Pediatrics, Keck School of Medicine, University of Southern California, LOS Angeles, USA; ${ }^{4}$ Specialist Children's and Young People's Services, East London NHS FT, London, UK

\subsection{6/archdischild-2020-rcpch.387}

Aim A neonatal m-health application (NeoTree) is being codesigned to support health care workers (HCWs) in lowincome countries (LICs). A key function is the digital implementation of evidence-based guidelines. Clinical support algorithms have been developed using best available national and international evidence and clinical judgement. We aimed to determine whether a panel of neonatal experts could address evidence gaps by reaching a consensus on four algorithms: neonatal sepsis, birth asphyxia, respiratory distress and hypothermia.

Methods This was a consensus-generating study using a twostep modified Delphi method. In round one, experts rated algorithm items and justified their responses. Items meeting consensus ( $>80 \%$ agreement) were included. Items not meeting consensus were excluded or only included following major edits or unchanged if they presented core elements of evidence-based guidelines. In round two, experts further rated items from round one that did not reach consensus.

Results Fourteen experts participated in round one, ten in round two. Nine were from high-income countries, five from
LICs. Experts included physicians and nurse practitioners with an average neonatal experience of 20 years, 12 in LICs. After two rounds, a consensus was reached on $52 \%$ of 84 items. Experts consistently stated that items must be consistent with local and WHO guidelines. An example of a key change in an algorithm included the management of transient tachypnoea of the newborn (TTN). The proposed NeoTree algorithm aimed to differentiate TTN from other respiratory conditions to avoid unnecessary antibiotic use. Expert consensus was that HCWs would be unable to appropriately distinguish and monitor patients with TTN. In the revised respiratory algorithm, all neonates with respiratory distress will be given antibiotics. As a result, the final algorithms included $53(62 \%)$ items.

Conclusion Four algorithms in a neonatal m-health treatment app were reviewed and refined by consensus expert opinion. Revisions to the NeoTree app were made in response to these findings and will be clinically validated in an imminent study. Expert discussion reinforced gaps in evidence in neonatal care in LIC, highlighting areas for future research.

\section{G450(P) SHORT TERM GLOBAL HEALTH PROJECT: A PILOT SCHEME IN SIERRA LEONE TO ENHANCE TRAINEE DEVELOPMENT}

${ }^{1}$ A Zahari, ${ }^{1} T$ Oliver, ${ }^{1} \mathrm{~N}$ Kennedy, ${ }^{2} \mathrm{R}$ Emeric, ${ }^{3} \mathrm{P}$ Turay, ${ }^{1} \mathrm{~V}$ Sawicki, ${ }^{4} \mathrm{P}$ Chahal, ${ }^{1,5} \mathrm{P}$ Chahal. ${ }^{1}$ Postgraduate Training, Health Education East Midlands, Leicester, UK; ${ }^{2}$ Public Health, University of Makeni, Makeni, Sierra Leone; ${ }^{3}$ Chief Medical Officer, Holy Spirit Hospital, Makeni, Sierra Leone; ${ }^{4}$ Co-founder, Help Madina, UK; ${ }^{5}$ Associate Professor, University of Nottingham, Nottingham, UK

\subsection{6/archdischild-2020-rcpch.388}

Introduction The NHS is experiencing declining number of doctors entering specialist training. Additionally, the BMA reports travel as the main reason for taking time out of training. The Global Health Qualitative Evaluation Project $(\mathrm{GH}-$ QEP) is an innovative pilot scheme that aims to address this. We believe opportunities to work abroad for a short term within a training program will appeal to those considering leaving the NHS or as an alternative to time out of program. It also builds skills and understanding, enhancing long term development as clinicians.

Design GH-QEP was a 4-week program based in Sierra Leone. Our team comprised of one paediatric and two GP trainees from the UK. Senior support was provided by UK and local consultants. The project consisted of qualitative evaluation of the Sierra Leone healthcare system post-Ebola through interviews of healthcare professionals and the public. The Ebola epidemic exposed an already stretched healthcare system within the country creating a climate of uncertainty. Significant effort implemented by the government and private organisations looked to rectify this. We received training in qualitative research methodology, and led the project design and implementation. Observing clinical practice in a variety of settings (hospital, rural feeding clinic, and district health unit) allowed hands-on experience of health issues in a challenging environment.

Outcome Undertaking qualitative evaluation is an opportunity not routinely provided in UK specialty training programs. Through patient interviews we learnt about the impact of government policies, education, local health beliefs and priorities of rural and urban population. It allowed us to reflect on the assumptions which shape our own understandings of health, 
illness and healthcare provision. The positive examples of individual leaders who had observed shortfalls and designed solutions to respond to the health needs of their local population inspired us to adopt similarly proactive approaches in our own clinical practice. Living and working in Sierra Leone developed our personal resilience and was emotionally impactful. Overall, a short-term program like GH-QEP not only benefits trainees personally and professionally, it might help increase specialty trainee application and retention. Furthermore, it could be expanded to incorporate a binational exchange program.

\section{G451(P) BLOOD TRANSFUSION SAFETY; KNOWLEDGE OF AMONG MEDICAL STAFFS PRE AND POST EDUCATIONAL INTERVENTION}

S Sahmoud, M El-Kalioby, E Ashry. Paediatrics, Suez Canal University, Ismailia, Egypt

10.1136/archdischild-2020-rcpch.389

The serious hazards of blood transfusion report showed that most of the errors reported in 2015 resulted from human factors and only $10 \%$ of them were not preventable, thus staff training on safe blood transfusion was strongly recommended. Aim To assess blood transfusion safety knowledge among medical staffs and how much it improves after intervention.

Methods A quasi-experimental study was conducted on 190 participants most of them were working on a tertiary nonteaching hospital for more than 8 years. A questionnaire was designed and validated through a pilot study after which all participants were invited to fill it pre and post educational intervention. The educational material has been prepared based on the WHO blood transfusion safety guidelines (WHO/EHT/10.05) in the form of leaflets and short power point presentations prepared by the researcher and reviewed by external experts in the field.

Results Near miss was identified by only half of the participants and around $78.3 \%, 63.2 \%$, and $60 \%$ of them correctly identified the indication of red blood cells, fresh frozen plasma, and platelet transfusion. These percentages were significantly improved post education. Only 50\% knew that it's not allowed to co-administrate drugs or IV fluids with the transfused blood and that rose to almost $80 \%$ after intervention. Consent information and correct patient identification were well known among most of the staff. Only $18.4 \%$ knew the pre transfusion screening protocol, which was increased to $85.8 \%$ post education. Almost $65.3 \%$ correctly responded to the transfusion reaction quiz with no marked change after intervention. Age and work experience were significant independent risk factors for poor knowledge of safety transfusion. Conclusion Transfusion safety knowledge needs further enhancement with more tailored training programs focusing on the topics that didn't show a significant change after our educational training such as the various transfusion reactions.

\section{G452(P) 'COUNTING THE UNCOUNTED': A DESCRIPTIVE STUDY OF THE DIAGNOSES PRESENTING TO A COMMUNITY PAEDIATRIC CLINIC IN ODISHA STATE, INDIA}

A Nye, M Cusack, P Marelli, E Norris, C Morris. Paediatric Clinic, Love the One India, Berhampur, Odisha, India

10.1136/archdischild-2020-rcpch.390
Introduction Odisha State had the third worst health index ranking among Indian states in 2017-2018. Relative to India as a whole, it has significantly worse infant mortality rate, under 5 mortality rate, and vaccination coverage. Although free public healthcare should be provided for those living below the poverty line, poor and vulnerable children are often denied medical treatment due to institutional bias.

Our charity provides free healthcare, childcare and education for poor and underprivileged children. The clinic provides primary health care for children enrolled in the charity's school and preschools; and Paediatric care for children with disabilities or chronic disease. A disproportionately high number have significant medical conditions, as they are unable to access quality health care via conventional means.

Aims To describe the epidemiology of our patients and the range of conditions seen.

Methods The notes of all Paediatric patients who attended the clinic from September 2018 to August 2019 were reviewed.

Results Over the year, there were 1008 patient encounters. $57 \%$ were by males. The age range was from 1 month to 15 years (median 6 years). 63\% of encounters were for management of acute illness, $11 \%$ were due to trauma, and $23 \%$ were related to chronic disease management. The most common diagnoses were lower respiratory tract infection, viral upper respiratory tract infection, tonsillitis, gastroenteritis and dental problems. Of the acute infections, it was presumed that $49 \%$ were bacterial, 39\% viral, 9\% parasitic and 5\% fungal. 'Tropical' infections encountered include typhoid, TB, dengue fever and HIV. Of the patient encounters that related to chronic diseases, the most common underlying diagnosis was cerebral palsy. Other significant long-term conditions include: sickle cell disease, thalassaemia, spina bifida, hydrocephalus, congenital heart disease, diabetes mellitus and hepatocellular carcinoma.

Conclusion Without this clinic, many of the children would have been unable to access healthcare, and may have experienced increased long-term morbidity or mortality. Children, including those with disabilities, are now able to access appropriate and prompt management of acute and chronic illness. We have demonstrated the impact a low-resource clinic can have, and hope to replicate this model across the state.

\section{G453(P) MODIFIED SPIKES, A NOVEL APPROACH TO COUNSELLING PARENTS OF PREMATURE BABIES}

ZG Abusalah. Neonatal Intensive Care Unit, Mediclinic City Hospital, Dubai, UAE

\subsection{6/archdischild-2020-rcpch.391}

Introduction Counselling of parents expecting a preterm baby is a challenging experience that requires special expertise and knowledge. Despite its importance, there is no standard approved approach to conduct it.

Aims

- To highlight the importance of effective communication with parents.

- To suggest a structured approach for counselling.

Methods The presentation will provide an overview of principles and contents of the counselling session. Although this has been the subject of several reviews and statements, there is a lack of a structured approach for conducting the consultation. 